\title{
Privacy Policy Images on Content Sharing Sites
}

\author{
Sakshi Datrange ${ }^{1}$, Swarali Lakade ${ }^{2}$, Nikita Kamble ${ }^{3}$, Prasad Kamble ${ }^{4}$, Prof. S. P. Godse ${ }^{5}$ \\ Student, Comp Dept, Stes Sinhgad Academy of Engineering Kondhwa, Pune, India ${ }^{1,2,3,4}$ \\ Guide, Comp Dept, Stes Sinhgad Academy of Engineering Kondhwa, Pune, India ${ }^{5}$
}

\begin{abstract}
Privacy of personal images is important for user acceptance. The media offer attractive means for interaction and communication, but also raise privacy and security concerns. Online social media such as My Space, or the Instagram, Facebook have experienced exponential growth in recent years. We analyze the impact of privacy concerns on image.
\end{abstract}

Keywords: Online Social Media, Image Privacy

\section{INTRODUCTION}

Images is a one of the key enablers of users' connectivity. Sharing takes place both among previously established groups of known people or social media. Most content sharing websites allow users to enter their privacy preferences. Unfortunately, recent studies have shown that users struggle to set up and maintain such privacy on image. One of the main reasons provided is that given the amount of shared image this process can be tedious and error prone. Therefore, many have acknowledged the need of policy recommendation systems which can assist users to easily and properly configure privacy. However, existing proposals for automating privacy appear to be inadequate to address the unique privacy needs of images due to the amount of information implicitly carried within images, and their relationship with the online environment wherein they are exposed.

In this paper, we propose an Privacy Policy on image. System which aims to provide users privacy on shared image user uploaded images, and factors in the following criteria that influence one's privacy of images:_ The impact of social media environment and personal characteristics. Social media context of users, such as their profile information with others may provide useful information regarding users' privacy preferences. For example, users interested in photography may like to share their photos with other photographers. Users who have several family members, friends, among their social contacts may share with them pictures related to family events or other. In light of these considerations, it is important to find the balancing point between the impact of social environment and users' individual characteristics in order to predict the policies that match each individual's needs opinions should be carefully considered. The role of image's content and metadata. In general, similar images often incur similar privacy preferences, especially when people appear in the images. We build the web base application in that user create the login after that they will share photo with their friends or other. With tag and permission.

Admin also create their profile. After that they will add user. And view the user posts. With tags and permission .with the help of algorithm it will post the image on application and this image can be view the permission authorized person are above 18 or below 18 .

\section{LITERATURE SURVEY}

\begin{tabular}{|l|l|l|l|}
\hline Sr.no & Title & year & Description \\
\hline & $\begin{array}{l}\text { "Imagined Communities: } \\
\text { Awareness, Information }\end{array}$ & $\begin{array}{l}\text { Sharing, and Privacy on the } \\
\text { Facebook Preproceedings } \\
\text { version." }\end{array}$ & $\begin{array}{l}\text { In this paper "Students living in the scholarship halls were written } \\
\text { up in early February for pictures on facebook.com that indicated a } \\
\text { party violating the scholarship halls alcohol policy" }\end{array}$ \\
1
\end{tabular}


Vol. 8, Issue 5, May 2019

\begin{tabular}{|c|c|c|c|}
\hline Sr.no & Title & year & Description \\
\hline 2 & $\begin{array}{l}\text { "iPrivacy: Image Privacy } \\
\text { Protection by Identifying } \\
\text { Sensitive Objects via Deep } \\
\text { Multi-Task Learning." }\end{array}$ & 2016 & $\begin{array}{l}\text { Therefore, we propose a new tool called iPrivacy which automates } \\
\text { the privacy setting process and releases the burden from users. } \\
\text { Specifically, our work consists of the following contributions: (a) } \\
\text { massive social images and their privacy setting, (b)leam more } \\
\text { representative deep CNNs and more discriminative tree classifier } \\
\text { over a visual tree, so that we can achieve fast and accurate detection } \\
\text { of large numbers of privacy-sensitive object classes; (c) automatic } \\
\text { recommendation of privacy settings for image sharing can be } \\
\text { achieved by detectint the underlying privacy-sensitive objects from } \\
\text { the images being shared, recognizing their classes, and identifying } \\
\text { their privacy settings; and (d) one simple solution for image privacy } \\
\text { protection is provided by blurring the privacy-sensitive objects } \\
\text { automatically. }\end{array}$ \\
\hline 3 & $\begin{array}{l}\text { "IMPROVING PRIVACY } \\
\text { IN JPEG IMAGES," }\end{array}$ & 2017 & $\begin{array}{l}\text { In this paper the massive distribution and sharing of images } \\
\text { increases the risk of privacylosses. There are several image formats, } \\
\text { but one of the most used is JPEG. To take care of this, the JPEG } \\
\text { standardization committee is promoting an activity on privacy and } \\
\text { security, in order to provide protection at image level. This paper } \\
\text { presents a mechanism to improve privacy in JPEG images by adding } \\
\text { privacy policies inside them and encrypting both policies and } \\
\text { images to perform access control based on those policies. Results of } \\
\text { a feasibility experiment are also described. Part of our contribution } \\
\text { is intended to be proposed for JPEG standardization. }\end{array}$ \\
\hline
\end{tabular}

\section{EXISTING SYSTEM}

In existing system creating privacy controls for social networks that are both expressive and usable is a major challenge. Lack of user understanding of privacy settings can lead to unwanted disclosure of private information and, in some cases, to material harm. Most users currently stick with their default, operator-chosen settings, such a system could dramatically increase the privacy protection that most users experience with minimal time investment

\section{PROPOSED SYSTEM}

In proposed system we analyse user and admin.

User: It will create own profile. User can enter their username and password after that this information is save in data base. User can create a group of friends. User will upload photo on that application with tags and provide authority for viewer like view, likes, comments, download, public/private and share.

The additional feature is below 18 user con not be view the image .or 18 above view the image asper the user privilege.

Admin: Admin also create own profile .In that they can enter their name and password. After that this information is save in data base.

It has authorities for add images to database and can view the users and images uploaded by user with privileges. User can search images for which they can give input as image. Image tags will be categorized based on gender and age of user. And accornadly user get result.

\section{SYSTEM ARCHITECTURE}

In this system architecture the main function is perform Admin and user.

Admin: To view the user images and upload by user privilege. And also add to Database with tags and Information.

User: Create profile and upload images with group .and user privilege the user can download the image.

This function can be perform in web base application. 
Following diagram is our system's architecture diagram:

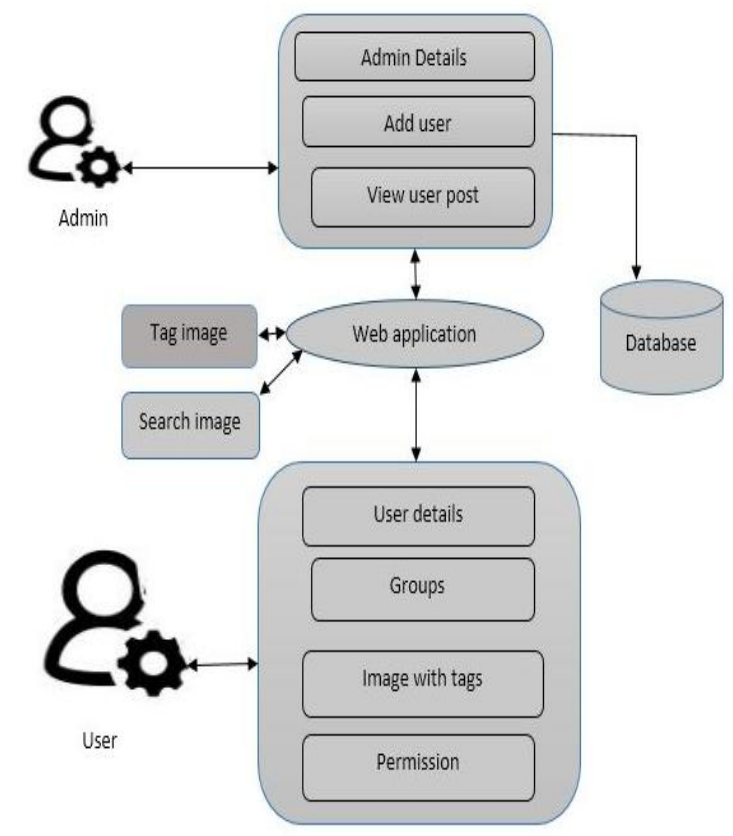

Figure 1: system architecture

\section{METHODOLOGIES}

The system will decide policy on its own for new users trying access to the images and thus the privacy of the images will be protected. The system will also cover the aspect of providing user with refine searching of images with bi-modal query approach.

In this work, we propose an Privacy Policy system to help users compose privacy settings for their images. In particular, we examine the role of image content and metadata as possible indicators of user's privacy preferences. We propose a two -level image classification framework to obtain image categories which may be associated with similar policies. Then, we develop a policy prediction algorithm to automatically generate a policy for each newly uploaded image.

Decision-making is regarded as the cognitive process resulting in the selection of a belief or a course of action among several alternative possibilities. Every decision-making process produces a final choice, which may or may not prompt action.

Decision-making is the process of identifying and choosing alternatives based on the values, preferences and beliefs of the decision-maker. The major part of decision-making involves the analysis of a finite set of alternatives described in terms of evaluative criteria. Then the task might be to rank these alternatives in terms of how attractive they are to the decision-maker(s) when all the criteria are considered simultaneously

\section{RELATED WORK}

Online media allow users to control the privacy set- tings of their shared content. Yet, the user finds it difficult to understand and correctly configure the offered access control policies. As a result, several studies have identified a serious mismatch between the desired and the actual privacy settings of content. This discrepancy motivated the development of mechanisms that aid users in selecting appropriate privacy settings. In the work of, for instance, the admin is focused on user posts and evaluated prediction models that make use of users' previous posts and profile preferences in order to suggest appropriate privacy settings for new posts. Despite achieving high performance, the admin noticed differences in user behaviors and concluded that personalized privacy models, were among the first to consider the problem of privacy-aware image classification. In their work, a large-scale user study was conducted asking participants to annotate a large number of publicly available Flickr photos as being either private or public. also attempted to solve a more complex privacy classification problem where three types of disclosure were defined for each image like view, comment, download and the task was to assign one of five privacy levels like as 'Only You', 'Family', 'Friends', 'Social Network', 'Everyone' to each type of disclosure. As in , their models captured only a generic perception of privacy. Differently from the majority of previous works, our paper highlights the limitations of generic 
Vol. 8, Issue 5, May 2019

image privacy classification models and proposes an effective personalization method. To the best of our knowledge, [4] is the only work that considers privacy classification of personal photos as we do here. While uses only metadatabased like location, time, etc. and simple visual features colors, edges, etc., we state-of-the-art CNN-based semantic visual features that facilitate comprehensible explanations of the classification outputs. Recently, evaluated the performance of deep features on again in the context of a privacy model and found that they yield remarkable improvements in performance compared to SIFT, GIST and user-assigned tag features. Moreover, the authors evaluated the performance of 'deep tag' features which are similar to the first level of semantic features that we extract here but did not exploit them for justifying the classifier's decisions.

VIII. RESULT

\section{Update Your Profile}

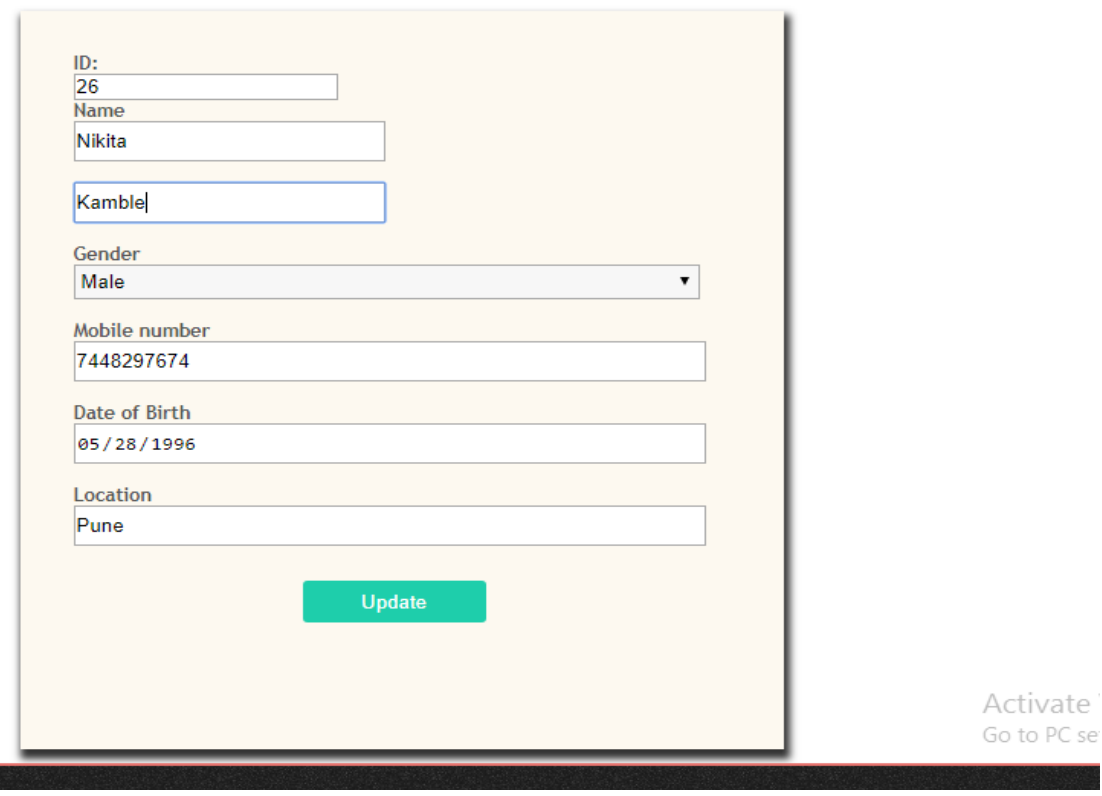

\section{User Authentication}

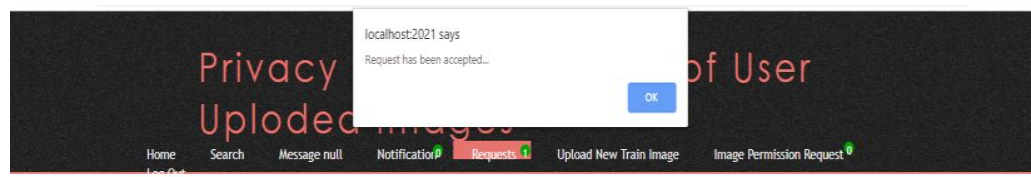

REQUESTS ARE:

goreparshut techbuzz@egmail com has Sent you friend requast $\mid$ ACCEPT $\mid \underline{\text { DENY }}$ 
Vol. 8, Issue 5, May 2019

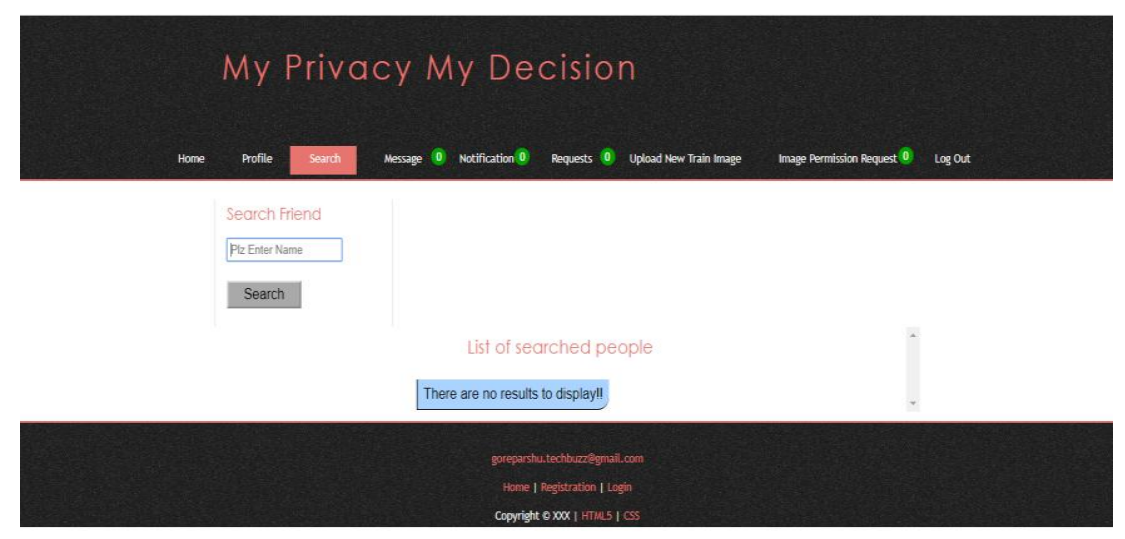

\section{CONCLUSION}

Photo sharing is a standout amongst the most prominent elements in online social networks,. We have proposed an Privacy Policy system that helps users automate the privacy policy settings for their uploaded images. We are using Decision making algorithm based on the information available for a given user. To prevent possible privacy leakage of a photo, we design a mechanism to enable each individual in a photo be aware of the posting activity and participate in the decision making on the photo posting. User choice the can be send photo above or below 18 user.

\section{REFERRENCES}

[1]. Imagined Communities: Awareness, Information Sharing, and Privacy on the Facebook Pre-proceedings version. Privacy Enhancing Technologies Workshop (PET), 2006 Alessandro Acquisti1 and Ralph Gross2.

[2]. iPrivacy: Image Privacy Protection by Identifying Sensitive Objects via Deep Multi-Task Learning IEEE TRANSACTIONS ON INFORMATION FORENSICS AND SECURITY, 2016.

[3]. Improving Privacy in Jpeg images Jaime Delgado, Silvia Llorente Distributed Multimedia Applications Group (DMAG) - Departament Arquitectura Computadors (DAC) Universitat Politècnica de Catalunya (UPC) · BarcelonaTECH C/Jordi Girona, 1-3, 08034 Barcelona.

[4]. Anna Cinzia Squicciarini, Dan Lin, Smitha Sundareswaran, and Joshua Wede, "Privacy Policy Inference of User-Uploaded Images on Content Sharing Sites", ieee transactions on knowledge and data engineering, vol. 27, no. 1, january 2015.

[5]. J. Bonneau, J. Anderson, and L. Church, "Privacy suites: Shared privacy for social networks," in Proc. Symp. Usable Privacy Security, 2009.

[6]. S. Ahern, D. Eckles, N. S. Good, S. King, M. Naaman, and R. Nair, "Over-exposed: Privacy patterns and considerations in online and mobile photo sharing," in Proc. Conf. Human Factors Comput. Syst., 2007, pp. 357-366.

[7]. A. Acquisti and R. Gross, "Imagined communities: Awareness, information sharing, and privacy on the facebook," in Proc. 6th Int. Conf. Privacy Enhancing Technol. Workshop, 2006, pp. 36-58.

[8]. A. Besmer and H. Lipford, "Tagged photos: Concerns, perceptions, and protections," in Proc. 27th Int. Conf. Extended Abstracts Human Factors Comput. Syst., 2009, pp. 4585-4590. 